\title{
A Treatment Combination of Peels, Oral Antioxidants, and Topical Therapy for Refractory Melasma: A Report of 4 Cases
}

This article was published in the following Dove Press journal: Clinical, Cosmetic and Investigational Dermatology

\author{
Jaime Piquero-Casals (D) \\ Corinne Granger $\mathbb{D}^{2}$ \\ Vanesa Piquero-Casals (1D ${ }^{1}$ \\ Aurora Garre ${ }^{2}$ \\ Juan Francisco Mir-Bonafé \\ 'Department of Aesthetic Dermatology \\ and Laser, Dermik, Clínica Dermatológica \\ Multidisciplinar, Barcelona, Spain; \\ ${ }^{2}$ Innovation and Development, ISDIN \\ Pharmaceutical Laboratories, Barcelona, \\ Spain
}

Background: Melasma is a difficult-to-treat, recurrent pigmentary disease. Combined therapy gives better, longer-lasting results.

Objective: To determine the clinical effects of a treatment protocol of trichloroacetic acid, phytic acid and ascorbic acid peel combined with oral antioxidant supplement and topical treatment for refractory melasma.

Patients and Methods: We present four cases of patients with melasma, who, despite multiple treatments including hydroquinone, showed no improvement. We initiated a 16week protocol involving 3 in-clinic peels ( 4 weeks apart) and a daily home treatment. The peels contained $30 \%$ trichloroacetic acid, $2 \%$ phytic acid, $8 \%$ L-ascorbic acid, Camellia sinensis leaf extract and Vitis vinifera seed extract. The home treatment was a depigmenting serum (4-butyl resorcinol, hydroxy-phenoxy propionic acid and niacinamide), a specific SPF50+ sunscreen, and an oral supplement (Polypodium leucotomos; green tea extract; Vitis vinifera; vitamins $\mathrm{C}, \mathrm{E}$, and $\mathrm{D}$; and carotenoids), all in the morning, and, at night, a compounded gel-cream ( $4 \%$ hydroquinone, $0.025 \%$ tretinoin and $1 \%$ hydrocortisone). After 16 weeks, the gel-cream was stopped; the rest of the regimen (topical and oral) was continued for 12 further weeks. Melasma was assessed using the melasma severity scale (MSS) before starting the protocol, and at 4 and 12 weeks after the last peel. Photographs were taken before treatment and at the last evaluation. Patients indicated their satisfaction on a 5-point scale.

Results: All patients had good tolerance to the procedures. Three showed an excellent (>75\%) improvement and one showed a good (50-75\%) improvement. All four were very satisfied. At follow-up (12 weeks after last peel), no patients had recurrence.

Conclusion: This protocol of trichloroacetic acid, phytic acid and ascorbic acid peel combined with an oral supplement and topical daily treatment is a viable treatment option for refractory melasma.

Keywords: trichloroacetic acid, phytic acid, ascorbic acid, peel, peelings, oral supplement, Polypodium leucotomos, topical treatment, melasma

\section{Introduction}

Melasma is one of the most frequently acquired hyperpigmentation disorders, with a prevalence of around 1 to $5 \%$ in the general population, or as high as $30 \%$ in specific populations such as in Latin America. ${ }^{1,2}$ It usually presents in middle-aged women as asymptomatic brown patches on the face, with symmetrical disposition and irregular borders. The disease is influenced by factors such as sun exposure (including visible light), genetic background, and female sex hormones. ${ }^{3}$
Correspondence: Jaime Piquero-Casals Carrer Escoles Pias 7. CP, Barcelona 08017, Spain

Email jaime.piquero@e-campus.uab.cat 
Initially considered a melanocyte dysfunction, its pathophysiology seems to be more complex, also involving keratinocytes, inflammatory cells, increased vascularization, abnormal elastic fibers and basement membrane disruption, leading some authors to consider it as a photoaging skin disease. ${ }^{4,5}$

Despite the existence of effective therapies, this condition remains a challenge for the dermatologist as the clinical history of the disease usually involves inconsistent therapeutic results and almost constant relapses.

The mainstay of melasma treatment remains prevention of exposure to sunlight and depigmenting topical agents. Photoprotection must include protection against blue light as its role in pigmentation disorders is now well recognized. ${ }^{6}$ Hydroquinone, a tyrosinase inhibitor, is the most used product with close to $40 \%$ of subjects having clearance following 12 weeks of twice-daily treatment at a concentration of $4 \%{ }^{7}$ When combined with corticosteroids and vit A derivatives, the triple combination led to at least a $75 \%$ improvement in $77 \%$ of subjects treated for 8 weeks. ${ }^{8}$

Chemical peels have also been proposed in the management of melasma but their use remains controversial because of the risk of skin irritation and post-inflammatory pigmentation and the lack of robust studies demonstrating the magnitude of the effect. Patients must, therefore, be carefully selected. Peels with trichloroacetic acid (TCA) are used in dermatology for the treatment of melasma, photo-aging and field cancerization. ${ }^{9,10}$

Some publications have reported on patients who, having not responded to traditional topical hydroquinone therapy, had an excellent response after 2 months of treatment when in-clinic monthly peelings were added to their treatment regimen. This demonstrated that, by removing excess layers of keratin, chemical peels can enhance or maximize the effect of hydroquinone or other depigmenting agents. ${ }^{11,12}$

More recently, systemic approaches for the treatment of melasma have been proposed. There is a growing interest in oral antioxidant supplements like extract of Polypodium leucotomos, green tea extract and vitamin C. ${ }^{13-15}$ These ingredients improve photoprotection by decreasing the production or limiting the effect of the oxidative stress. Their effect in melasma is, however, poorly documented. It has been demonstrated that TCA associated with antioxidants such as ascorbic acid is superior to TCA alone and helps maintain the response to melasma therapy. ${ }^{16}$
We present four cases of patients with a long history of melasma resistant to multiple treatments, for whom a protocol with domiciliary treatment combined with monthly chemical peels gave good responses and encouraged us to use such a treatment combination when applicable in future.

\section{Case Presentation}

The patients were four women with a clinical diagnosis of melasma confirmed with Wood lamp and with a history of more than five years with the disease and multiple infructuous treatments with kojic acid, hydroquinone, cysteamine and topical retinoids. All patients signed informed consent forms. They also signed forms giving their consent for the use of case details and images for scientific purposes.

We designed a protocol involving a peeling treatment performed in the clinic along with a daily home treatment. The patients started the combination home treatment four weeks before the first peeling, to prepare the skin and avoid undesirable effects. The home treatment consisted of, each morning, a depigmenting serum containing a combination of 4-butyl resorcinol, hydroxy-phenoxy propionic acid and niacinamide (Pigment Expert ${ }^{\mathbb{B}}$ ISDIN ampoules), a specific SPF50+ sunscreen, and an oral supplement containing $480 \mathrm{mg}$ of Polypodium leucotomos; green tea extract; Vitis vinifera; vitamins $\mathrm{C}, \mathrm{E}$, and $\mathrm{D}$; and carotenoids (Sunisdin ${ }^{\circledR}$ ) and, at night, a triple combination compounded medication with $4 \%$ hydroquinone, $0.025 \%$ tretinoin and $1 \%$ hydrocortisone in a gel-cream for a duration of 16 weeks. The in-clinic treatment consisted of a chemical peel every 4 weeks (three sessions). The peels contained 30\% trichloroacetic acid, $2 \%$ phytic acid, 8\% L-ascorbic acid, Camellia sinensis leaf extract and Vitis vinifera seed extract (Isdinceuticals BRA peeling $\left.{ }^{\circledR}\right)$. At the end of the protocol, the hydroquinone was stopped and the rest of the topical regime and the oral nutricosmetic were continued for 12 further weeks, when the final follow-up was conducted. All cosmetic treatments used, are approved for use in Europe, and the protocol did not involve any invasive or unauthorized products.

Treatment outcomes (melasma severity) were assessed clinically using the melasma severity scale (MSS), before starting the treatment regimen and 4 and 12 weeks after the third and final peeling session. The MSS is a four-point grading system: 0 , melasma almost equivalent to surrounding normal skin or with minimal residual pigmentation; 1 , mild, slightly darker than surrounding normal skin; 2, 
moderate, moderately darker than surrounding normal skin; 3, severe, markedly darker than surrounding normal skin.

Digital photographs were taken before treatment and at the final evaluation and assessed visually by the investigator. The percentage improvement on these photographs was assessed using the following four-point scale: weak (0-25\% improvement), moderate (25-50\% improvement), good (50-75\% improvement), excellent ( $>75 \%$ improvement). The treatment was considered effective overall when the improvement was greater than $50 \%$.

Patients indicated their satisfaction with the treatment on a 5-point scale (very satisfied, satisfied, neither satisfied nor unsatisfied, unsatisfied, or very unsatisfied).

\section{Results}

Prior to treatment, three of the patients had grade 3 (severe) melasma and one had grade 2 (moderate) melasma.

At the end of the treatment (week 16) all four patients had grade 1 (mild) melasma.
All patients showed a cosmetic improvement and good tolerance to the procedures and topical products. In the photographic evaluation, three patients had an excellent improvement (more than 75\% improvement) and one had a good improvement (50-75\% improvement) (Figure 1). All the patients reported they were very satisfied with the combination treatment and the results. No adverse events were reported.

At follow-up, at 12 weeks after the last peel, all four patients had a maintained therapeutic response with scores stable from the previous assessment (3 excellent and 1 good improvement).

\section{Discussion}

Melasma is a frustrating pigmentation disorder for patients and physicians alike. A combination treatment regimen can achieve better results without recurrences and without raising safety concerns. ${ }^{4}$ Nutricosmetics containing antioxidants and anti-aging properties may be useful and are simple to use as a part of treatment. ${ }^{14,15}$ Depigmenting
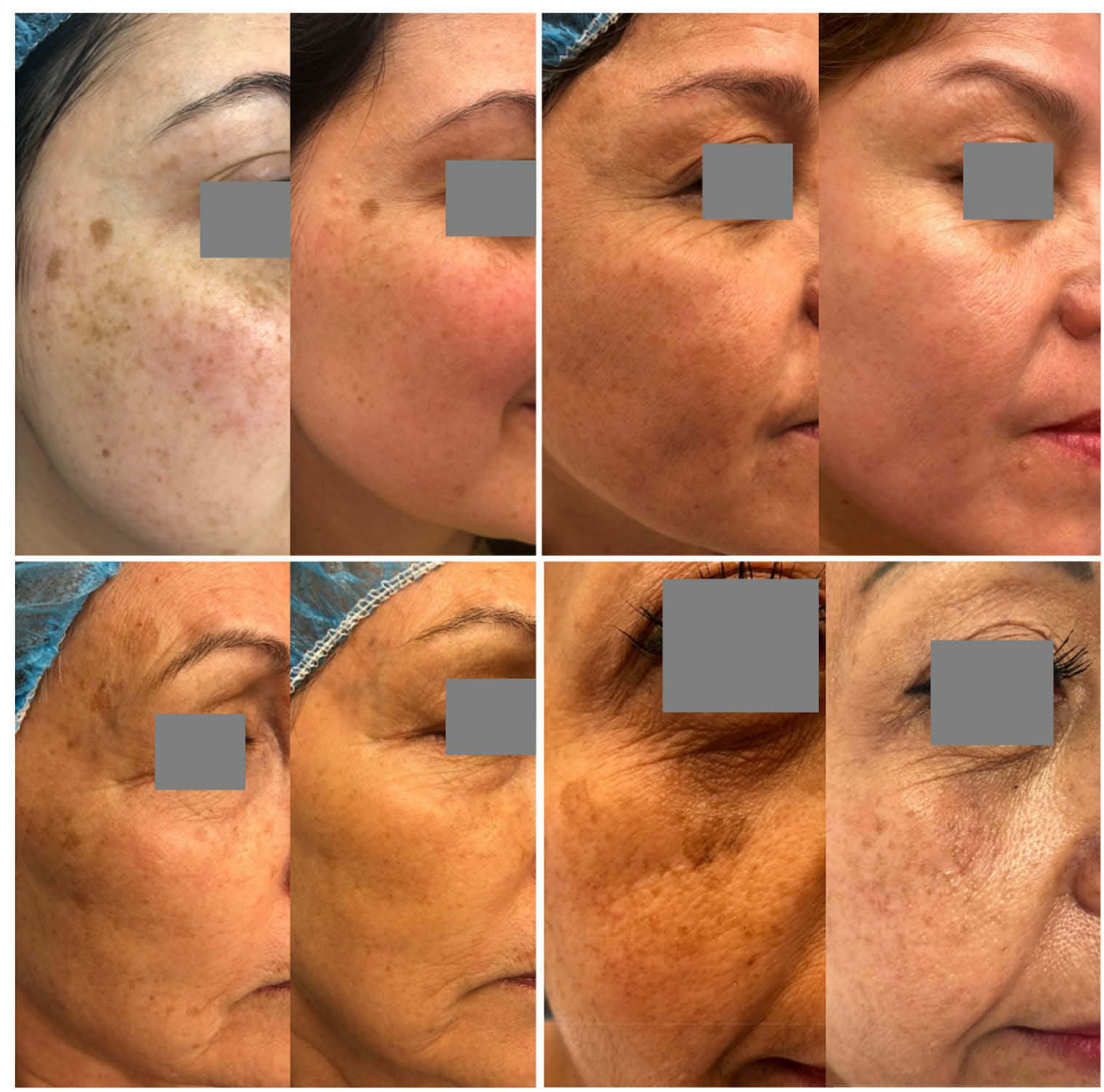

Figure I Four patients before and after treatment, 4 weeks after the last peeling. Patients consented to use of images. 
peelings used in association with trichloroacetic acid, ascorbic and phytic acids represent an effective option. ${ }^{9,10}$ One study concluded that the combination of $20 \%$ TCA with 5\% ascorbic acid was a highly effective and well-tolerated therapy with low recurrence of melasma.16 Another more recent study using chemical peels with TCA alone or combined with glycolic acid showed a significant improvement in melasma in both. ${ }^{17}$ Daily treatment with mild depigmenting agents has been shown to help maintain the treatment effects of peeling. ${ }^{18}$

It is important in this population to use a broadspectrum sunscreen with high SPF and good protection against UVA and visible light, which are known aggravating factors of pigmented lesions and melasma. ${ }^{4}$ Recent findings revealed that UVA exposure induced CPD generation via chemoexcitation in melanocytes hours after exposure. ${ }^{19}$ Another recent study determined that the use of antioxidants provided protection against UVA-induced delayed CPD formation by enhancing ROS scavenging activity and melanogenesis inhibition. ${ }^{20}$ These are important considerations in the pathophysiology of melasma: therapeutic options should be adapted to target all the associated factors and not just hyperpigmentation.

The four patients presented here had a long history of topical hydroquinone, cysteamine and kojic acid treatment without improvement. However, no oral or topical antioxidant was used with the previous treatments. When treated with this protocol of a daily oral and topical therapy in combination with in-clinic peeling, a clinical improvement in melasma was observed.

Three of the patients had an excellent improvement (more than $75 \%$ improvement) and one had a good improvement (50-75\% improvement). All the patients were very satisfied with the combination treatment and the results. No adverse events were reported. During the follow-up period (to 12 weeks post-peeling) all patients maintained a good therapeutic response of improvement of hyperpigmentation with no recurrence.

With this treatment protocol, we observed very good results with no relapse at the 12-week follow-up, with continued daily home therapy. Some limitations of the study design must be taken into account. Firstly, the assessment of photographs to determine clinical severity of MSS did not use a standardized light source or software analysis, but rather relied on investigator evaluation. In addition, our study was designed to assess a combination treatment - this means that we cannot identify any one of the treatment components as having a stronger or weaker treatment effect. This series of cases could be extended in future studies to a greater number of cases and with a longer follow-up, taking into account the recurrent nature of this disease. With this therapeutic combination, we were able to achieve very good results with no relapse after 12 weeks of continued therapy.

\section{Conclusions}

Trichloroacetic acid, phytic acid and ascorbic acid peel combined with an oral supplement and topical home treatment is a suitable treatment option for refractory melasma. The combined treatment used here was well tolerated by patients, safe, and highly effective. This combination regimen for melasma needs to be explored in future studies with greater patient numbers.

\section{Disclosure}

Dr Jaime Piquero-Casals reports grants from ISDIN during the conduct of the study and grants from ISDIN outside the submitted work. Dr Corinne Granger reports grants from ISDIN during the conduct of the study. Dr Aurora Garre Contreras reports personal fees from ISDIN outside the submitted work. The authors report no other conflicts of interest in this work.

\section{References}

1. Pichardo R, Vallejos Q, Feldman SR, et al. The prevalence of melasma and its association with quality of life in adult male Latino migrant workers. Int J Dermatol. 2009;48:22-26. doi:10.1111/ijd.2009.48. issue-1

2. Werlinger KD. Prevalence of self-diagnosed melasma among premenopausal Latino women in Dallas and Fort Worth, Tex. Arch Dermatol. 2007;143:423-431. doi:10.1001/archderm.143.3.424

3. Kwon SH, Na JI, Choi JY, Park KC. Melasma: updates and perspectives. Exp Dermatol. 2019;28(6):704-708. doi:10.1111/exd.13 844

4. Passeron T, Picardo M. Melasma, a photoaging disorder. Pigment Cell Melanoma Res. 2018;31(4):461-465. doi:10.1111/pcmr.12684

5. Rajanala S, Maymone MBC, Vashi NA. Melasma pathogenesis: a review of the latest research, pathological findings, and investigational therapies. Dermatol Online J. 2019;25(10).

6. Regazzetti C, Sormani L, Debayle D, et al. Melanocytes sense blue light and regulate pigmentation through opsin-3. J Invest Dermatol. 2018;138(1):171-178. doi:10.1016/j.jid.2017.07.833

7. Ennes SBP, Paschoalick RC, De Avelar Alchorne MM. A double-blind comparative placebo-controlled study of the efficacy and tolerability of $4 \%$ hydroquinone as a depigmenting agent in melasma. J Dermatol Treat. 2000;11:173-179. doi:10.1080/09546630050517333

8. Taylor SC, Torok H, Jones T, et al. Efficacy and safety of a new triple combination agent for the treatment of facial melasma. Cutis. 2003;72 (1):67-72.

9. Abdel-meguid AM, Taha EA, Ismail SA. Combined Jessner solution and trichloroacetic acid versus trichloroacetic acid alone in the treatment of melasma in dark-skinned patients. Dermatol Surg. 2017;43 (5):651-656. doi:10.1097/DSS.0000000000001036 
10. Truchuelo M, Cerdá P, Fernández LF. Chemical peeling: a useful tool in the office. Actas Dermosifiliogr. 2017;108(4):315-322. doi:10.10 16/j.ad.2016.09.014

11. Mashiko T, Oka A, Osawa E, Koshima I. A deceptively simple solution for refractory melasma: glycolic acid peels and hydroquinone at home. Plast Reconstr Surg Glob Open. 2017;5(5):e1335. doi:10.1097/GOX.0000000000001335

12. Chaudhary S, Dayal S. Efficacy of combination of glycolic acid peeling with topical regimen in treatment of melasma. J Drugs Dermatol. 2013;12(10):1149-1153.

13. Bala HR, Lee S, Wong C, Pandya AG, Rodrigues M. Oral tranexamic acid for the treatment of melasma: a review. Dermatol Surg. 2018;44 (6):814-825. doi:10.1097/DSS.0000000000001518

14. Choudhry SZ, Bhatia N, Ceilley R, et al. Role of oral Polypodium leucotomos extract in dermatologic diseases: a review of the literature. J Drugs Dermatol. 2014;13(2):148-153.

15. Thitimuta S, Pithayanukul P, Nithitanakool S, Bavovada R, Leanpolchareanchai J, Saparpakorn P. Camellia sinensis L. Extract and its potential beneficial effects in antioxidant, anti-inflammatory, anti-hepatotoxic, and anti-tyrosinase activities. Molecules. 2017;22 (3):401. doi:10.3390/molecules22030401
16. Dayal S, Sahu P, Yadav M, Jain VK. Clinical efficacy and safety on combining 20\% trichloroacetic acid peel with topical 5\% ascorbic acid for melasma. J Clin Diagn Res. 2017;11(9):WC08-WC11. doi:10. 7860/JCDR/2017/26078.10685

17. Garg S, Thami GP, Bhalla M, Kaur J, Kumar A. Comparative efficacy of a $35 \%$ glycolic acid peel alone or in combination with a $10 \%$ and $20 \%$ trichloroacetic acid spot peel for melasma: a randomized control trial. Dermatol Surg. 2019;45(11):1394-1400. doi:10.1097/DSS.0000000000001964

18. McKesey J, Tovar-garza A, Pandya AG. Melasma treatment: an evidence-based review. Am J Clin Dermatol. 2019. doi:10.1007/ s40257-019-00488-w

19. Premi S, Wallisch S, Mano CM, et al. Photochemistry. chemiexcitation of melanin derivatives induces DNA photoproducts long after UV exposure. Science. 2015;347(6224):842-847. doi:10.1126/science.1256 022

20. Yim S, Lee J, Jo H, et al. Chrysanthemum morifolium extract and Ascorbic Acid-2-Glucoside (AA2G) blend inhibits UVA-induced delayed cyclobutane pyrimidine dimer (CPD) production in melanocytes. Clin Cosmet Investig Dermatol. 2019;12:823-832. doi: $10.2147 /$ CCID.S223802

\section{Publish your work in this journal}

Clinical, Cosmetic and Investigational Dermatology is an international, peer-reviewed, open access, online journal that focuses on the latest clinical and experimental research in all aspects of skin disease and cosmetic interventions. This journal is indexed on CAS
The manuscript management system is completely online and includes a very quick and fair peer-review system, which is all easy to use. Visit http://www.dovepress.com/testimonials.php to read real quotes from published authors. 